\title{
The stress analysis of a shear wall with matrix displacement method using rectangular finite element
}

\author{
Mustafa Ergün*1 (iD), Şevket Ateş ${ }^{2}$ iD \\ ${ }^{1}$ Bayburt University, Department of Civil Engineering, Bayburt, Turkey \\ ${ }^{2}$ Karadeniz Technical University, Department of Civil Engineering, Trabzon, Turkey
}

\begin{abstract}
The aim in this study is to numerically present some characteristic features of the rectangular finite element using the matrix displacement method and to show the utility of this element in plane stress problems compared to the finite element method. The paper consisted of three parts. In the first part, all of the finite element formulation steps from choosing the convenient coordinate system to creating element stiffness matrix are presented respectively. In the second part of the study, a static finite element analysis of the shear wall is also made by ANSYS Mechanical APDL. In the final part, the results (displacements, strains and stresses) obtained from the previous parts are compared with each other by the help of tables and graphics. The results show that this method is effective and preferable for the stress analysis of shell structures. Further studies should be conducted in order to indicate the efficiency of the matrix displacement method for the solution of different types of plane stress problems using different finite elements.
\end{abstract}

\section{Keywords}

Plane stress problem; Matrix displacement method; Rectangular finite element

Received: 12 January 2021; Accepted: 16 February 2021

ISSN: 2630-5763 (online) (C) 2021 Golden Light Publishing All rights reserved.

\section{Introduction}

The finite element method (FEM) was created because of the need to solve complex elasticity and structural problems in civil and aeronautical engineering. Hrennikoff [1] and Courant [2] made significant contributions to the development of FEM with their studies. While Courant divided the domain into finite triangular elements to solve second order elliptic partial different equations, Hrennikoff discretized the domain by using a lattice analogy. In 1950s, this method came into use for the solution of problems in applied sciences and engineering area, particularly for stress analysis of aircraft bodies. In later years, FEM obtained its real impetus by available open-source finite element software programs like finite element program SAP IV made by UC Berkeley. The method has been commonly used since 1950s and 1960s for the numerical modeling and analysis of physical systems in a wide variety of engineering disciplines. In the civil engineering area, the studies on linear and nonlinear analyses of structures can be given as examples of FEM applications [3-9].

The basic idea of FEM is that the main problem is divided into sub-problems to create discrete problems that are more understandable and easily solvable; thus, the solution of the main problem consists of combining the solutions of the discrete

* Corresponding author

Email: mustafaergun@bayburt.edu.tr 
sub-problems. In engineering applications, approximate solutions at an acceptable level are adequate rather than exact results for some complicated problems. Since exact results for these types of problems are considered impossible due to their complexity, approximate solutions are the only options for design. When mathematical tools are inadequate for obtaining precise or even approximate result, FEM is the only method that can be used.

Two-dimensional elastic problems were the first successful examples of the application of the finite element method [10]. Different analytical methods were used to analyze shell structures; however, studies of behavior of this type of structure have significantly increased since the development of FEM. Studies have been conducted by researchers for years to develop new finite elements that properly present behaviors of shell structures. Lee and Bathe [11] developed a simple method to design isotropic triangular shell finite elements based on the mixed interpolation of tensorial components (MITC) approach. The proposed method is mechanically clear as well as simple and effective. Numerical tests are carried out using selected MITC elements. Proposed elements show good performance for test elements with different thickness. Mousa and Tayeh [12] developed a new triangular finite element called SBTREIR for general plane elasticity. This element has three degrees of freedom at each of the three corner nodes. The performance of the new element was compared with a well-known constant strain triangle (CST) element. The new finite element showed good behavior in the elasticity theory. Also, it had fewer discontinuities in the corner stresses than the CST. A new three-node triangular shell element was developed using discrete Kirchhoff theory and a mixed method [13]. The goal of this study was to conduct stress analyses of shell structures using the free mesh method, an improved 8 -node shell element for the analysis of plates and shells [14]. The finite element, based on refined first-order shear deformation theory, was further improved by the combined use of the assumed natural strain method. They analyzed the influence of the shell element with the different patterns of sampling points for interpolating different components of strains. Rebiai and Belounar [15] developed a new simple and efficient four-node quadrilateral membrane finite element with drilling rotation. Wang and Sun [16] developed a new 4node quadrilateral flat shell element for geometrically nonlinear analyses of thin and moderately thick laminated shell structures. The flat shell element was constructed by combining a quadrilateral area coordinate method (QAC) based membrane element AGQ6-II, and a Timoshenko beam function (TBF) method-based shear deformable plate bending element ARS-Q12. Numerical results showed that the present shell element had an excellent numerical performance for the test examples and was applicable to stiffened plates. Ergun and Ates [17] obtained the stress distribution of a shear wall with matrix displacement method using CST. The solution obtained from this method showed excellent agreements with the results of the analytical method.

Different types of computer software coded by finite element methods have been commonly used to analyze shell structures. However, engineers usually focus on structural behaviors such as stresses, strains, displacements, and internal forces to design these types of structures. They do not concern themselves with the logic of computer software programs, so, in this study, we tried to present the general structure of the rectangular finite element, one of the most popular element types of FEM, in three stages. In the first part, all of the finite element formulation steps including choosing the appropriate coordinate system and numbering, idealization of the system with rectangular finite elements, developing the structural and loading characteristics of the structure in matrix form, and creating element stiffness matrixes and the matrix algebra analysis for displacements and stresses of the structure, was presented respectively. In the second part of the study, a static finite element analysis of the shear wall was conducted [18]. In the third and final part, the results (displacements, strains, and stresses) 
obtained from the parts one and two were compared with each other with the help of tables and graphics. Consequently, the basic aim in our study is to present numerically some characteristic features of a rectangular finite element using the matrix displacement method and to show the utility of this element in plane stress problems by comparison with finite element method, so the next parts of the paper include studies carried out for this purpose.

\section{The finite element formulation of the rectangular element}

The rectangular finite element (RFE) is very useful in solving many stress problems wherever the domain can be discretized into rectangles. In this paper, RFE is used to create a finite element model of a plane stress problem. The steps for the development of the element stiffness matrix are described below.

\subsection{Choosing the appropriate coordinate system and numbering}

There are two displacement parameters in both $X$ and $Y$ directions for each node of RFE as shown in Fig. 1.

\subsection{Choosing the displacement function that} defines displacements at every point of the element

The displacement function can be written for each direction using Pascal's triangle as may be seen below.
1

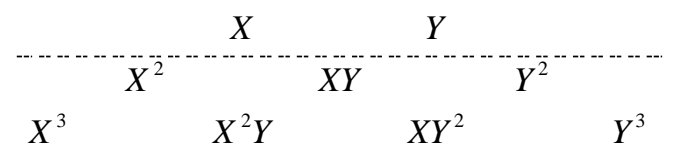

$U_{X}=\alpha_{1}+\alpha_{2} X+\alpha_{3} Y+\alpha_{4} X Y$

$U_{Y}=\alpha_{5}+\alpha_{6} X+\alpha_{7} Y+\alpha_{8} X Y$

where $\alpha_{1}, \alpha_{2}, \alpha_{3}, \alpha_{4}, \alpha_{5}, \alpha_{6}, \alpha_{7}, \alpha_{8}$ are adjustable parameters. It is assumed that the displacements vary linearly within the element in this notation.

\subsection{The strain $\{\boldsymbol{\varepsilon}(\mathbf{X}, \mathbf{Y})\}$ - displacement}

$\{\mathbf{U}(\mathbf{X}, \mathbf{Y})\}$ relationship at any point of the element

Using the strain-displacement equations, the internal strain can be written as

$$
\varepsilon_{X}=\frac{\partial U_{X}}{\partial X}=\alpha_{2}+\alpha_{4} Y
$$

$\varepsilon_{Y}=\frac{\partial U_{Y}}{\partial Y}=\alpha_{7}+\alpha_{8} X$

$\gamma_{X Y}=\frac{\partial U_{X}}{\partial Y}+\frac{\partial U_{Y}}{\partial X}=\alpha_{3}+\alpha_{4} X+\alpha_{6}+\alpha_{8} Y$

$\{\varepsilon(X, Y)\}=\left\{\begin{array}{l}\varepsilon_{X} \\ \varepsilon_{Y} \\ \gamma_{X Y}\end{array}\right\}=\left\{\begin{array}{l}\alpha_{2}+\alpha_{4} Y \\ \alpha_{7}+\alpha_{8} X \\ \alpha_{3}+\alpha_{4} X+\alpha_{6}+\alpha_{8} Y\end{array}\right\}$

$\{\varepsilon(X, Y)\}=[B]\{U\}$

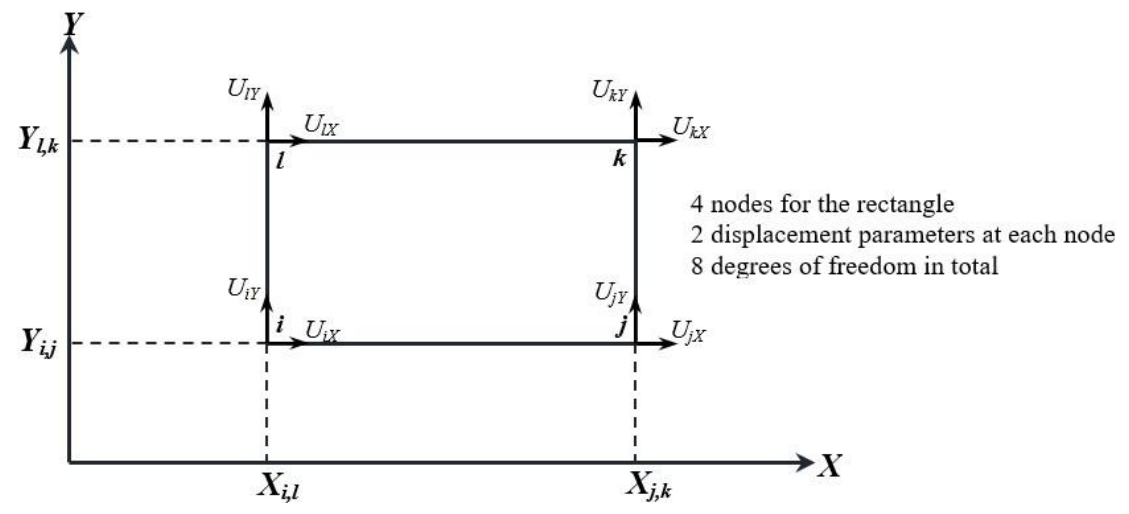

Fig. 1. Rectangular element and its coordinates 
where $[B]$ matrix is created as below:

$[B]=\left[\begin{array}{cccccccc}-\frac{1}{a}+\frac{Y}{a b} & 0 & \frac{1}{a}-\frac{Y}{a b} & 0 & \frac{Y}{a b} & 0 & -\frac{Y}{a b} & 0 \\ 0 & -\frac{1}{b}+\frac{X}{a b} & 0 & -\frac{X}{a b} & 0 & \frac{X}{a b} & 0 & \frac{1}{b}-\frac{X}{a b} \\ -\frac{1}{b}+\frac{X}{a b} & -\frac{1}{a}+\frac{Y}{a b} & -\frac{X}{a b} & \frac{1}{a}-\frac{Y}{a b} & \frac{X}{a b} & \frac{Y}{a b} & \frac{1}{b}-\frac{X}{a b} & -\frac{Y}{a b}\end{array}\right]$

\subsection{The stress $\{\boldsymbol{\sigma}(\mathbf{X}, \mathbf{Y})\}$-strain $\{\boldsymbol{\varepsilon}(\mathbf{X}, \mathbf{Y})\}$

$$
W_{\text {ext }}=\left\{U^{*}\right\}^{T}\{F\}
$$
relationship at any point of the element

Using the stress-strain equations, the internal stress can be written as

$$
\{\sigma(X, Y)\}=[D]\{\varepsilon(X, Y)\}
$$

where $[\mathrm{D}]$ is material matrix given by

$$
[D]=\left[\begin{array}{ccc}
d_{11} & d_{12} & 0 \\
d_{21} & d_{22} & 0 \\
0 & 0 & d_{33}
\end{array}\right]
$$

where $\{F\}$ and $\left\{U^{*}\right\}$ denote nodal forces and nodal virtual displacements respectively.

$$
W_{\text {int }}=\int_{0}^{v}\left\{\varepsilon(X, Y)^{*}\right\}^{T}\{\sigma(X, Y)\} d v
$$

where $\varepsilon(X, Y)^{*}$ is strain caused an arbitrary virtual displacement, and $\sigma(X, Y)$ is real stress in the element.

If Eqs. (6)-(7) are written in their own places at Elements of this matrix are given in Table 1.

\subsection{The relationship between nodal displacements and nodal forces}

According to the virtual work principle, the total work done by all forces in a system in static equilibrium is zero for a set of infinitesimally small displacements. The equation below must be satisfied in order to fulfill this principle.

$$
W_{\text {ext }}=W_{\text {int }}
$$

where $W_{\text {ext }}$ and $W_{\text {int }}$ are strain works done by external forces and internal forces respectively.

Table 1. Constants for plane stress and plane strain problems [19]

\begin{tabular}{ccc}
\hline Variable & Plane Stress & Plane Strain \\
\hline$d_{11}=d_{22}$ & $\frac{E}{\left(1-v^{2}\right)}$ & $\frac{E(1-v)}{(1+v)(1-2 v)}$ \\
$d_{12=d_{21}}$ & $\frac{E v}{\left(1-v^{2}\right)}$ & $\frac{E v}{(1+v)(1-2 v)}$ \\
$d_{33}$ & $\frac{E}{2(1+v)}$ & $\frac{E}{2(1+v)}$
\end{tabular}


If Eqs. (9)-(12) are written in their own places at Eq. (8), the Eq. (13) is obtained as below:

$$
\begin{aligned}
& \left\{U^{*}\right\}^{T}\{F\}=\int_{v}\left\{U^{*}\right\}^{T}[B]^{T}[D][B]\{U\} d v \\
& \{F\}=\int_{v}\{B\}^{T}[B][D]\{U\} d v
\end{aligned}
$$

The relationship between force and displacement is known as

$$
\{F\}=[K]\{U\}
$$

When Eqs. (13)-(14) are compared with each other, stiffness matrix of RFE is calculated as below:

$$
[K]=\int_{v}[B]^{T}[D][B] d v
$$

where $d v=t d x d y$, in this case stiffness of RFE can be written again as below:

$$
[K]=t \int_{Y=0}^{Y=b} \int_{X=0}^{X=a}[B]^{T}[D][B] d x d y
$$

where $\mathrm{t}$ is thickness of RFE.

Finally, if $[B]^{T},[D]$ and $[B]$ are written in their own places at Eq. (15), and integral is calculated, the stiffness matrix $(8 \times 8)$ can be obtained, which is given in Appendix.

\section{Stress analysis of shear wall with numerical methods (Matrix Displacement Method and ANSYS Mechanical APDL)}

First, the static analysis of the shear wall under external loads are performed by the help of the matrix displacement method. Discretized finite element model of the considered shear wall is shown in Fig. 2. For the material, $E=2.1 \times 10^{7}$ $\mathrm{kN} / \mathrm{m}^{2}$, and $v=0.2$. Thickness of the wall is taken to be $t=20 \mathrm{~cm}$.

The stiffness matrix of the shear wall, and the strain and stress functions for both elements are, respectively, given in Appendix. The nodal displacements and stresses obtained from this approach are given in Fig. 3.

Second, the static analysis of the shear wall under external loads are performed by the help of the finite element method using ANSYS Mechanical APDL. Nodal displacements, strains and stresses obtained from this approach are given in Tables 2-6 and Fig. 4.

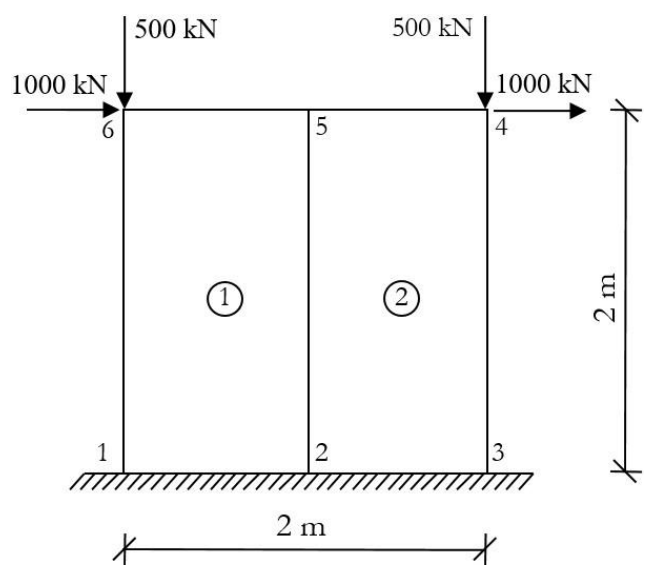

Fig. 2. Discretized finite element model, nodes and loading case of the shear wall

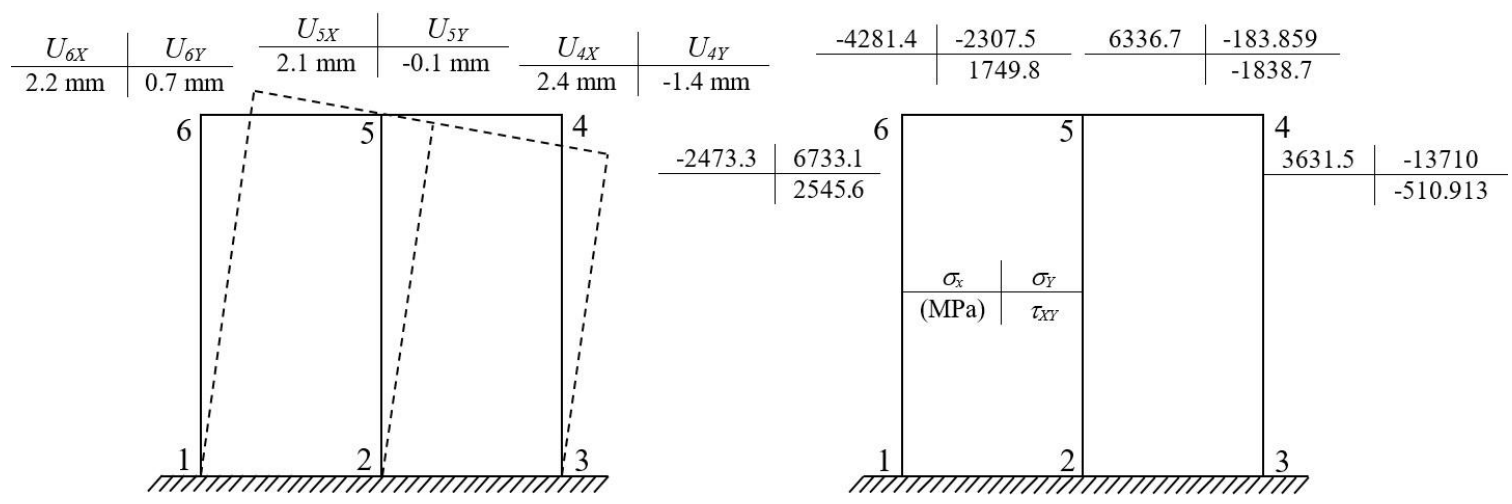

Fig. 3. Deformed shape, nodal displacements and nodal stresses 
Table 2. Nodal displacement values of the shear wall under concentrated loads

\begin{tabular}{cccc}
\hline Node & $U_{X}(\mathrm{~m})$ & $U_{Y}(\mathrm{~m})$ & $U_{Z}(\mathrm{~m})$ \\
\hline 1 & 0.00000000 & 0.00000000 & 0.00000000 \\
2 & 0.00000000 & 0.00000000 & 0.00000000 \\
3 & 0.00000000 & 0.00000000 & 0.00000000 \\
4 & 0.00236600 & -0.00137490 & 0.00000000 \\
5 & 0.00205310 & -0.00013821 & 0.00000000 \\
6 & 0.00223500 & 0.00068836 & 0.00000000 \\
\hline
\end{tabular}

Table 3. Elastic strain values of the Element 1 under concentrated loads

\begin{tabular}{ccccc}
\hline Node & $\varepsilon_{X}$ & $\varepsilon_{Y}$ & $\varepsilon_{Z}$ & $\gamma_{X Y}$ \\
\hline 1 & 0.000000000 & 0.000344180 & -0.000086044 & 0.001117500 \\
2 & 0.000000000 & -0.000069104 & 0.000017276 & 0.001026500 \\
5 & -0.000181940 & -0.000069104 & 0.000062762 & 0.000199990 \\
6 & -0.000181940 & 0.000344180 & -0.000040558 & 0.000290960 \\
\hline
\end{tabular}

Table 4. Elastic strain values of the Element 2 under concentrated loads

\begin{tabular}{ccccc}
\hline Node & $\varepsilon_{X}$ & $\varepsilon_{Y}$ & $\varepsilon_{Z}$ & $\gamma_{X Y}$ \\
\hline 2 & 0.00000000 & -0.000069104 & 0.000017276 & 0.001026500 \\
3 & 0.00000000 & -0.000687430 & 0.000171860 & 0.001178300 \\
4 & 0.00030352 & -0.000687430 & 0.000095978 & -0.000058341 \\
5 & 0.00030352 & -0.000069104 & -0.000058604 & -0.000210100 \\
\hline
\end{tabular}

Table 5. Stress values of the Element 1 under concentrated loads

\begin{tabular}{ccccc}
\hline Node & $\sigma_{X}\left(\mathrm{kN} / \mathrm{m}^{2}\right)$ & $\sigma_{Y}\left(\mathrm{kN} / \mathrm{m}^{2}\right)$ & $\sigma_{Z}\left(\mathrm{kN} / \mathrm{m}^{2}\right)$ & $\tau_{X Y}\left(\mathrm{kN} / \mathrm{m}^{2}\right)$ \\
\hline 1 & 1505.8000 & 7528.9000 & 0.0000 & 9778.3000 \\
2 & -302.3300 & -1511.6000 & 0.0000 & 8982.3000 \\
5 & -4282.3000 & -2307.6000 & 0.0000 & 1749.9000 \\
6 & -2474.2000 & 6732.9000 & 0.0000 & 2545.9000 \\
\hline
\end{tabular}

Table 6. Stress values of the Element 2 under concentrated loads

\begin{tabular}{crrcc}
\hline Node & $\sigma_{X}\left(\mathrm{kN} / \mathrm{m}^{2}\right)$ & $\sigma_{Y}\left(\mathrm{kN} / \mathrm{m}^{2}\right)$ & $\sigma_{Z}\left(\mathrm{kN} / \mathrm{m}^{2}\right)$ & $\tau_{X Y}\left(\mathrm{kN} / \mathrm{m}^{2}\right)$ \\
\hline 2 & -302.3300 & -1511.6000 & 0.0000 & 8982.3000 \\
3 & -3007.5000 & -15037.0000 & 0.0000 & 10310.0000 \\
4 & 3632.0000 & -13710.0000 & 0.0000 & -510.4800 \\
5 & 6337.1000 & -183.7500 & 0.0000 & -1838.4000 \\
\hline
\end{tabular}



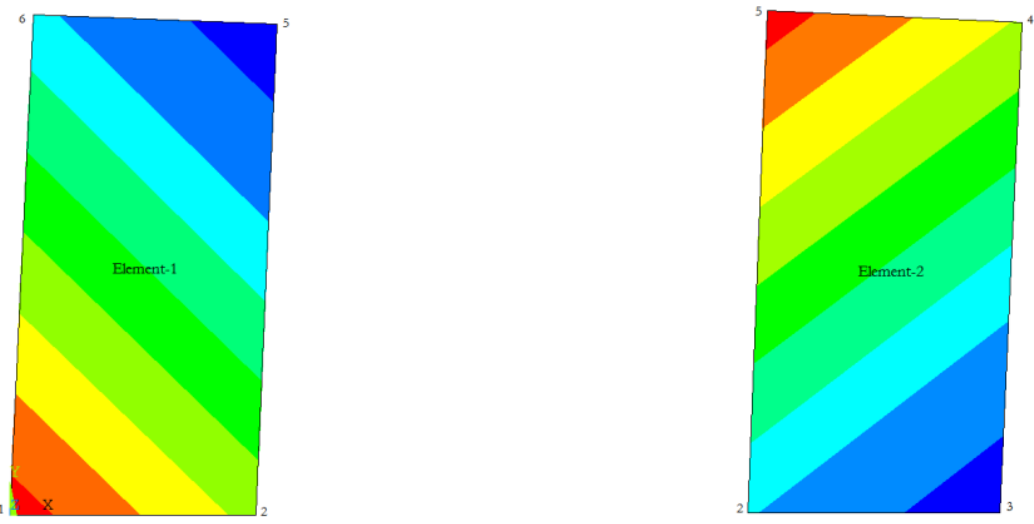

a) Element 1

b). Element 2

Fig. 4. Stress contour diagram of Element 1 and Element 2 under concentrated loads

\section{Results}

The results obtained from the analysis made by using both methods on the shear wall are compared with each other and given in the Tables 7-8.

The basic aim of our study is to numerically present some characteristic features of the rectangular finite element using the matrix displacement method and show the utility of this element in plane stress problems by comparison with the finite element method. The results given in Tables 7 and 8 show that this method is effective and preferable for the stress analysis of shell structures.

Table 7. Nodal stress values obtained from both approaches

\begin{tabular}{|c|c|c|c|}
\hline & $\mathrm{kN} / \mathrm{m}^{2}$ & Matrix Displacement Method & FEM (ANSYS Mechanical APDL) \\
\hline \multirow{12}{*}{ 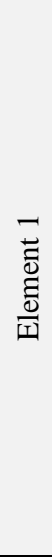 } & $\sigma_{1 X}$ & 1505.800 & 1505.800 \\
\hline & $\sigma_{1 Y}$ & 7528.900 & 7528.900 \\
\hline & $\tau_{1 X Y}$ & 9778.100 & 9778.300 \\
\hline & $\sigma_{2 X}$ & -302.334 & -302.330 \\
\hline & $\sigma_{2 Y}$ & -1511.700 & -1511.600 \\
\hline & $\tau_{2 X Y}$ & 8982.300 & 8982.300 \\
\hline & $\sigma_{5 X}$ & -4281.400 & -4282.300 \\
\hline & $\sigma_{5 Y}$ & -2307.500 & -2307.600 \\
\hline & $\tau_{5 X Y}$ & 1749.800 & 1749.900 \\
\hline & $\sigma_{6 X}$ & -2473.300 & -2474.200 \\
\hline & $\sigma_{6 Y}$ & 6733.100 & 6732.900 \\
\hline & $\tau_{6 X Y}$ & 2545.600 & 2545.900 \\
\hline \multirow{8}{*}{ 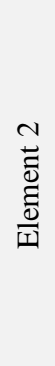 } & $\sigma_{2 X}$ & -302.334 & -302.330 \\
\hline & $\sigma_{2 Y}$ & -1511.700 & -1511.600 \\
\hline & $\tau_{2 X Y}$ & 8982.300 & 8982.300 \\
\hline & $\sigma_{3 X}$ & -3007.600 & -3007.500 \\
\hline & $\sigma_{3 Y}$ & -15038.000 & -15037.000 \\
\hline & $\tau_{3 X Y}$ & 10310.000 & 10310.000 \\
\hline & $\sigma_{4 X}$ & 3631.500 & 3632.000 \\
\hline & $\sigma_{4 Y}$ & -13710.000 & -13710.000 \\
\hline
\end{tabular}


Table 7. Continued

\begin{tabular}{|c|c|c|c|}
\hline & $\mathrm{kN} / \mathrm{m}^{2}$ & Matrix Displacement Method & FEM (ANSYS Mechanical APDL) \\
\hline \multirow{4}{*}{ 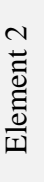 } & $\tau_{4 X Y}$ & -510.913 & -510.480 \\
\hline & $\sigma_{5 X}$ & 6336.700 & 6337.100 \\
\hline & $\sigma_{5 Y}$ & -183.859 & -183.750 \\
\hline & $\tau_{5 X Y}$ & -1838.700 & -1838.400 \\
\hline
\end{tabular}

Table 8. Nodal displacement obtained from both approaches

\begin{tabular}{|c|c|c|c|}
\hline & $\mathrm{m}$ & Matrix Displacement Method & (FEM) ANSYS Mechanical APDL \\
\hline \multirow{8}{*}{ 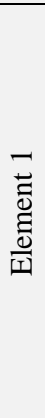 } & $U_{1 X}$ & 0 & 0 \\
\hline & $U_{1 Y}$ & 0 & 0 \\
\hline & $U_{2 X}$ & 0 & 0 \\
\hline & $U_{2 Y}$ & 0 & 0 \\
\hline & $U_{5 X}$ & 0.00210000 & 0.00205310 \\
\hline & $U_{5 Y}$ & -0.00010000 & -0.00013821 \\
\hline & $U_{6 X}$ & 0.00220000 & 0.00223500 \\
\hline & $U_{6 Y}$ & 0.00070000 & 0.00068836 \\
\hline \multirow{8}{*}{ 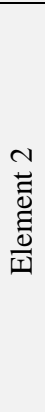 } & $U_{2 X}$ & 0 & 0 \\
\hline & $U_{2 Y}$ & 0 & 0 \\
\hline & $U_{3 X}$ & 0 & 0 \\
\hline & $U_{3 Y}$ & 0 & 0 \\
\hline & $U_{4 X}$ & 0.00240000 & 0.00235660 \\
\hline & $U_{4 Y}$ & -0.00140000 & -0.00137490 \\
\hline & $U_{5 X}$ & 0.00210000 & 0.00205310 \\
\hline & $U_{5 Y}$ & -0.00010000 & -0.00013821 \\
\hline
\end{tabular}

\section{Conclusions}

In this paper, we present the characteristic features of the rectangular finite element using the matrix displacement method and show utility of this element in plane stress problems by comparison with the finite element method. A shear wall used as an example of a plane stress problem is discretized into rectangular finite elements. The stress distribution of the shear wall is obtained by using the matrix displacement method and ANSYS Mechanical APDL.

From the results of both numerical and analytic studies, the following observations can be made:

- The stress values of the Element-1 and Element2 under concentrated loads obtained by using matrix displacement method show excellent agreements with the results of ANSYS Mechanical APDL.
- The strain values of Element-1 and Element-2 obtained from matrix displacement method overlap those obtained from ANSYS Mechanical APDL.

- The nodal displacement values of the shear wall obtained from both numerical and analytic methods are almost the same.

The results show that the proposed method is effective and preferable for the stress analysis of shells. Further studies should be conducted in order to indicate the efficiency of the matrix displacement method for the solution of different types of plane stress problems using different finite elements.

\section{Declaration of conflicting interests}

The author(s) declared no potential conflicts of interest with respect to the research, authorship, and/or publication of this article. 


\section{References}

[1] Hrennikoff A (1941) Solution of problems of elasticity by the framework method. Journal of Applied Mechanics 8(4):169-175.

[2] Courant R (1943) Variational methods for the solution of problems of equilibrium and vibrations. Bulletin of the American Mathematical Society 49:1-23.

[3] Akin JE. Applications and Implementation of Finite Element Methods. Academic Press, 1984.

[4] Hutton D. Fundamentals of Finite Elements Analysis, McGraw-Hill Co., New York, 2004.

[5] Zienkiewicz OC, Taylor RL. The Finite Element Method for Solid and Structural Mechanics. Butter worth-Heinemann, 2005.

[6] Long YQ, Cen S, Long ZF. Advanced Finite Element Methods in Structural Engineering. Springer-Verlag GmbH Berlin Heidelberg \& Tsinghua University Press, Beijing, 2009.

[7] Jain AK. Advanced Structural Analysis, Nem Chand \& Bros, 2009.

[8] Yun HD, Hwang JH, Kim MY, Choi SH, Park WS, Kim KS (2020) Seismic performance of RC frame structures strengthened by HPFRCC walls. Structural Engineering and Mechanics 75(3):389399.

[9] Heydari M, Behnamfar F, Zibasokhan H (2018) A macro-model for nonlinear analysis of $3 \mathrm{~d}$ reinforced concrete shear walls. International Journal of Engineering 31(2):220-227.

[10] Turner MJ, Clough RW, Martin HC, Topp LJ (1956) Stiffness and deflection analysis of complex structures. Journal of Aeronautical Sciences 23:805-823.
[11] Lee PS, Bathe KJ (2004) Development of isotropic triangular shell finite elements, MITC. Computers and Structures 82:945-962.

[12] Mousa AI, Tayeh SM (2004) A triangular finite element for plane elasticity with in plane rotation. Journal of the Islamic University of Gaza 12(1):8395.

[13] Yagawa G, Miyamura $T$ (2005) Three-node triangular shell element using mixed formulation and its implementation by free mesh method. Computers and Structures 83:2066-2076.

[14] Han SC, Kanok-Nukulchai W, Lee WH (2011) A refined finite element for first-order plate and shell analysis. Structural Engineering and Mechanics 40(2):191-213.

[15] Rebiai C, Belounar L (2014) An effective quadrilateral membrane finite element based on the strain approach. Measurement 50:263-269.

[16] Wang Z, Sun Q (2014) Corotational nonlinear analyses of laminated shell structures using a 4node quadrilateral flat shell element with drilling stiffness. Acta Mechanica Sinica 30(3):418-429.

[17] Ergun M, Ates S (2015) The stress analysis of a shear wall with matrix displacement method. Structural Engineering and Mechanics 53(2):205226.

[18] ANSYS 2015. Swanson Analysis System, Pennsylvania, USA.

[19] Timoshenko SP, Goodier JN. Theory of Elasticity. McGraw-Hill, New York, 1971. 


\section{Appendix}

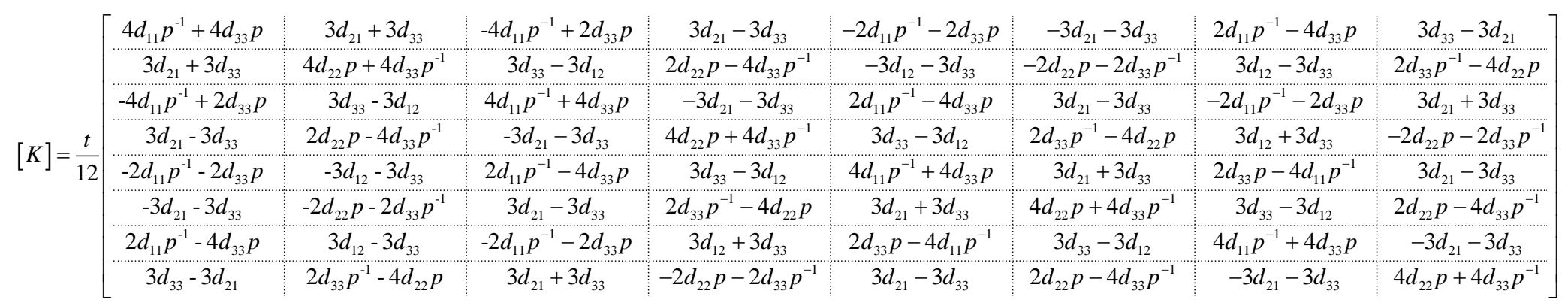

$[K]=\frac{0.2}{12}\left[\begin{array}{c:c:c:c:c:c}192,500,000 & 39,375,000 & -166,250,000 & -13,125,000 & 0 & 0 \\ 39,375,000 & 113,750,000 & 13,125,000 & -48,125,000 & 0 & 0 \\ \hdashline-166,250,000 & 13,125,000 & 385,000,000 & 0 & -166,250,000 & -13,125,000 \\ \hdashline-1,3125,000 & -48,125,000 & 0 & 227,500,000 & 13,125,000 & -48,125,000 \\ \hdashline 0 & 0 & -166,250,000 & 13,125,000 & 192,500,000 & -39,375,000 \\ \hdashline 0 & 0 & -13,125,000 & -48,125,000 & -39,375,000 & 113,750,000\end{array}\right]$

Strain and stress expressions for the element 1 ,
$\varepsilon_{x}=(-y / 20000)-(1 / 20000)$
$\sigma_{x}=(-1750 x)-(4375 y / 4)-(875 / 2)$
$\varepsilon_{y}=(3 / 20000)-(x / 2500)$
$\sigma_{y}=(6125 / 2)-(875 y / 4)-(8750 x)$
$\gamma_{x y}=(27 / 40000)-(y / 2500)-(x / 20000)$
$\tau_{x y}=(23625 / 4)-(3500 y)-(875 x / 2)$

and for the element 2 ,
$\varepsilon_{x}=(3 y / 20000)+(3 / 20000)$
$\sigma_{x}=(13125 y / 4)-(11375 x / 4)+(13125 / 8)$
$\varepsilon_{y}=(-13 x / 20000)-(3 / 8000)$
$\sigma_{y}=(2625 y / 4)-(56875 x / 4)-(60375 / 8)$
$\gamma_{x y}=(3 x / 20000)-(13 y / 20000)+(19 / 40000)$
$\tau_{x y}=(2625 x / 2)-(11375 y / 2)+(16625 / 4)$ 